\section{Test-rest reliability of mechanical and thermal stimuli for sensory testing in dogs with chronic, osteoarthritis-associated pain}

\section{David Knazovicky', Beth Case', Lyndy Harden², Duncan Lascelles ${ }^{1,2}$}

Comparative Pain Research Laboratory, Department of Clinical Sciences, College of Veterinary Medicine, North Carolina State University, Raleigh, NC, USA

Center for Comparative Medicine and Translational Research, Department of Clinical Sciences, College of Veterinary Medicine, North Carolina State University, Raleigh, NC, USA

Center for Pain Research and Innovation, UNC School of Dentistry, Chapel Hill, NC, USA

Quantitative sensory testing (QST) has become a common approach to assess the functional changes in the somatosensory/nociceptive system resulting from the presence of a chronic pain state such as Osteoarthritis (OA). The aim of this study was to evaluate test-retest reliability of mechanical and thermal threshold devices in client-owned dogs with osteoarthritis-associated pain.

Twenty five client-owned dogs (12 males castrated, 13 females spayed, $8.8 \pm 2.9$ years old and $31.8 \pm 8.0 \mathrm{~kg}$ ) with OA primarily affecting the hind limbs (hip and/or stifle) were tested on two occasions, one week apart (day 0; day 7). An electronic von Frey (EVF) (0.5 mm diameter tip) and a pressure algometer (PA) (3 mm diameter tip) were applied as ramped stimuli. A thermal probe (TP) (13 mm diameter) was applied as a hot and cold fixed stimulus, $49{ }^{\circ} \mathrm{C}$ and $0{ }^{\circ} \mathrm{C}$ respectively. Both hind limbs were tested at 3 sites (pre limb) in randomized order: OA-affected joint (periarticular sensitivity), cranial tibial muscle (muscle sensitivity) and dorsal metatarsal region (remote sensitivity). Differences between left and right hind limb and sensory thresholds between visits were evaluated using a non-parametric test (Wilcoxon signed rank test).

There was no significant difference between left and right hind limbs for any modality and at any site. Significantly lower thresholds were obtained on day 7 for the EVF at the affected, tibial and remote sites $(p=0.004, p<0.0001, p<0.0001$, respectively), and for the $P A$ at the tibial site $(p=0.027)$. Significantly higher TP-heat thresholds were obtained on day 7 at affected and tibial sites ( $p=0.044, p=0.001$, respectively).

Our data demonstrate the variability of somatosensory thresholds over time in dogs with OA in contrast to previous data in normal dogs. Understanding and controlling for this variability will be crucial if these tests are to be used to assess somatosensory functional changes associated with paininduced neuroplasticity.

Funding source: Boehringer Ingelheim Animal Health $\mathrm{GmbH}$ and Comparative Pain Research Laboratory

\section{Human Factors: factoring humans in to patient care}

\section{Mark Turner}

Safety in Practice Ltd, Kent, UK

Human Factors is a growing field and the focus of considerable attention in 'high risk' industries. Managers and strategists have identified the positive impact that a coherent human factors policy can have on an organisation's exposure to risk.

In the medical professions the subject of Human Factors examines the way workers interact with machines, work spaces, as well as each other, and how this affects patient safety.

The N.H.S. has only recently integrated human factors into staff training modules but it now forms a significant element. The health service has adopted this approach after studying the aviation industry, which has spent significant resources quantifying the role people play in safety.
Research has suggested that there are many parallels between flight cockpits and operating theatres. In both communication and healthy team dynamics are important in the prevention of accidents. Also, similarities have been identified in the way pilots and doctors conduct themselves at work.

Human Factors has highlighted the role leadership plays. Leaders need to foster an 'open' culture in which workers feel able to discuss mistakes and offer feedback, such that the overall 'system' is protected from sources of error.

This presentation summarizes research which forms the cornerstone of human factors training in the human health sector. It will re-visit the essential elements of the subject and emphasize those most pertinent to patient safety. Simple strategies by which we might improve in areas such as personal risk management and communication will be described. And it seeks to draw attention to the way in which apparently small incidents in the course of a working day can contribute to an adverse event, according to Reason's 'Swiss Cheese model' of accident causation. Above all it asks us to consider that by working more closely as a team, safety and the quality of care can be quite radically enhanced. 